Florian Morath

\title{
Volunteering and the Strategic Value of Ignorance
}

Max Planck Institute for Intellectual Property,

Competition and Tax Law

SP || $2010-17$

November 2010 
Florian Morath, Volunteering and the Strategic Value of Ignorance, Discussion Paper SP II 2010 - 17,

Wissenschaftszentrum Berlin, 2010.

Wissenschaftszentrum Berlin für Sozialforschung $\mathrm{gGmbH}$, Reichpietschufer 50, 10785 Berlin, Germany, Tel. (030) 25491 - 0 Internet: www.wzb.eu 


\title{
Volunteering and the Strategic Value of Ignorance
}

\author{
by Florian Morath *
}

Private provision of public goods often takes place as a war of attrition: individuals wait until someone else volunteers and provides the good. After a certain time period, however, one individual may be randomly selected. If the individuals are uncertain about their cost of provision, but can find out about this cost ahead of the volunteering game, a strategic value is attached to the information, and individuals may prefer not to learn their cost of provision. If the time horizon is sufficiently short, in equilibrium only one individual may acquire information about his cost. For a long time horizon, acquiring information is strictly dominant. The time limit is an important instrument in influencing the efficiency of the volunteering game.

Keywords: War of attrition, volunteering, discrete public goods, asymmetric information, information acquisition

JEL classification: H41, D44, D82, D83

\section{ZUSAMMENFASSUNG}

\section{Volunteering and the Strategic Value of Ignorance}

Die private Bereitstellung öffentlicher Güter ähnelt häufig einem "Zermürbungskrieg": Die Beteiligten warten, bis sich jemand anderes freiwillig meldet und das öffentliche Gut bereitstellt. Nach einer gewissen Zeitperiode des Wartens kann jedoch ein Beteiligter zufällig dazu bestimmt werden, die Bereitstellung zu übernehmen. Wenn die Beteiligten ihre Bereitstellungskosten nicht genau kennen, sich aber vor dem Bereitstellungsspiel Information über ihre Kosten beschaffen können, dann kommt dieser Information ein strategischer Wert zu; die Beteiligten könnten es vorziehen, ihre Bereitstellungskosten nicht genau zu kennen. Wenn der Zeithorizont des Bereitstellungsspiels hinreichend kurz ist, entscheidet sich im Gleichgewicht lediglich ein Beteiligter, Information zu akquirieren. Bei einem längeren Zeithorizont ist es eine strikt dominante Strategie, sich Information zu beschaffen. Der Zeithorizont stellt ein wichtiges Instrument zur Beeinflussung der Effizienz des Bereitstellungsspiels dar.

\footnotetext{
I thank Kai Konrad, Michael Michael, and Johannes Münster for valuable comments. Financial support by the German Research Foundation (DFG) through grant SFB/TR 15 is gratefully acknowledged.
} 


\section{Introduction}

Dragon-slaying and ballroom dancing are two famous examples ${ }^{1}$ for the provision of a public good that induces a positive value for a certain group of individuals. One of the individuals, however, has to pay some cost in order to provide the public good. Such situations are often best described by a war of attrition: one volunteer is needed for a certain task, and everyone prefers someone else to volunteer first and bear the cost of provision. Typically, there is a disutility or waiting cost attached to the time until a volunteer is found. In this paper, we study the individuals' incentives to obtain information about their own cost of provision of the public good prior to a volunteering game or war of attrition.

Wars of attrition are used to model a large number of applications from different fields. Besides dragon-slaying, many unpleasant situations like intervening in a fight, calling the police in case of a fire or crime, household chores, fights between animals, or market exit exhibit properties of wars of attrition. ${ }^{2}$ Organizations typically rely on the voluntary performance of a large number of tasks. These tasks may have to be performed repeatedly, and the cost of performing the task may then be well-known. But often the individuals don't know exactly how costly volunteering will turn out to be. They may, for instance, only have a guess about the time involved in chairing a university department or organizing a conference, but can acquire information about this expenditure of time.

In many companies or institutions, staff meetings take place on a regular basis and are used to allocate tasks to individuals. Before volunteering to perform a task, employees typically have the possibility to find out about their cost of performing this task, and they can do so by asking questions and collecting information. The question, however, is what impact information acquisition has on the volunteering game and whether individuals benefit from information acquisition. If such information acquisition can be observed by the other individuals - for instance when employees ask questions - there is a strategic value attached to the information: it can be used

\footnotetext{
${ }^{1}$ Cf. Bliss and Nalebuff (1984).

${ }^{2}$ Many more examples are given, e.g., by Bilodeau and Slivinski (1996), LaCasse et al. (2002), or Otsubo and Rapoport (2008).
} 
to commit to a certain behavior in the war of attrition. Similarly, on an international level, when governments have to decide whether to provide an international public good, they can engage experts to provide them with a better estimate of the cost of provision. But when such investments in information are observable by other players, investments in information obtain a strategic character. These examples have in common that the players cannot wait an infinite amount of time before volunteering, but that there is a time limit on their decision to concede.

We analyze the individuals' incentives to acquire information about their cost of provision of a public good in a two-stage game with two individuals. In the first stage, the individuals can obtain information about their cost of provision. In order to focus on the strategic considerations, we assume that the information is available at zero cost. Whether or not an individual decided to find out about his cost can be observed by the rival before the volunteering game starts. The information that an individual has obtained, however, is only privately known to this individual. In the second stage, a volunteering game or war of attrition takes place: the individuals simultaneously choose a maximum waiting time after which they provide the public good, given that nobody else has volunteered before. The waiting time until the public good is provided involves a direct cost. As described above, individuals may not be able to wait for an infinite amount of time; therefore, we impose a finite time horizon after which one of the individuals is randomly chosen to pay for the provision. At some point in time, the dragon may itself decide to attack, or, in the context of a firm, one employee will be selected by the team leader to perform the task.

As we will show, the equilibrium of the volunteering game and the incentives to learn the own cost of provision crucially depend on the length of the time horizon. For a long time horizon, both individuals prefer to find out about their cost of provision. If the time horizon of the volunteering game is sufficiently short, individuals without information about their provision cost prefer a random selection when the time limit is reached to an early concession. As a consequence, an individual who found out that his cost would be low may prefer to concede immediately. Therefore, not knowing the own cost of provision can be advantageous in the volunteering game. For a sufficiently 
short time horizon, there are two asymmetric equilibria where one individual finds out about his cost and the other does not, and one symmetric equilibrium where both individuals randomize their decision whether to learn their cost. The choice of the time horizon is an important instrument in influencing the efficiency of the volunteering game.

The literature on wars of attrition has its origin in applications in biology, modeling fights between animals (e.g., Maynard Smith 1974, Riley 1980). Further important applications are industrial competition and market exit (Fudenberg and Tirole 1986, Ghemawat and Nalebuff 1985, 1990). The seminal paper that studies the private provision of a public good as a war of attrition is Bliss and Nalebuff (1984). In their setup, the players are privately informed of their cost of provision, and the equilibrium is efficient in the sense that the player with the lowest cost provides the public good. The provision of multiple public goods in the framework of a war of attrition is analyzed by LaCasse et al. (2002) for the case of complete information, and by Sahuguet (2006) in an environment with private information. ${ }^{3}$ Bishop and Cannings (1978), Hendricks et al. (1988), Bilodeau and Slivinski (1996), and Myatt (2005) study models that exhibit a finite time horizon. We add to this literature by studying the effects of information on the individuals' concession times in the private provision game, and the resulting incentives (not) to become informed. The strategic considerations involved in the decision on information are similar to the strategic aspects identified in different settings such as principal-agent relationships (e.g. Crémer 1995, Kessler 1998): by remaining uninformed, individuals precommit to a certain behavior in the subsequent interaction. ${ }^{4}$

Closely related to this paper is work that considers information in auctions.

\footnotetext{
${ }^{3}$ Further papers considering wars of attrition with privately informed players are Bulow and Klemperer (1999), who analyze the case of multiple prizes, and Krishna and Morgan (1997), who study the case of affiliated signals. Amann and Leininger (1996) consider a general class of all-pay auctions with private information; the same class of all-pay auctions is analyzed in Riley (1999) for the case of complete information. Che and Gale (1998) study first-price all-pay auctions with caps on bidding which are similar to the finite time horizon of the volunteering game assumed here.

${ }^{4}$ In the context of global warming, Morath (2010) analyzes investments in information in a standard model of private provision of a continuous public good; the strategic effects that are present in this paper, however, are driven by the assumption that other countries can observe what a country has learned.
} 
Whereas the war of attrition is, in fact, a second-price all-pay auction, Morath and Münster (2010) study information acquisition in a first-price all-pay auction, but in their setup, there is no purely strategic value of remaining uninformed. In the context of winner-pay auctions, incentives to acquire information when decisions are observable have been shown to depend on the exact auction format and on whether information is about a private or a common value. An early contribution studying the value of information is Milgrom and Weber (1982); recent work includes Hernando-Veciana (2009), Larson (2009), and Hernando-Veciana and Tröge (2010).

The next section describes the setup of the model. We analyze in Section 3 the three different situations that may arise in the volunteering game: no individual has private information about his provision cost, only one individual is informed, or both individuals are informed about their cost of provision. In Section 4, we consider the incentives for information acquisition in a $2 \times 2$ game defined by the continuation payoffs in the volunteering game, and we discuss some implications from a designer's perspective. Section 5 assesses the robustness of our results. Finally, Section 6 concludes. All proofs are in the appendix.

\section{Setup}

Consider the following game with two individuals, 1 and 2. One of the two individuals has to provide a public good of fixed quantity. (We assume that the contribution that is needed for the provision is indivisible.) The individuals differ with respect to their cost of provision, denoted by $c_{1}$ and $c_{2}$. These cost parameters $c_{1}$ and $c_{2}$ are independent draws from a probability distribution that is common knowledge and assumed to be a discrete function with

$$
c_{i} \in\left\{c_{L}, c_{H}\right\}, \quad 0<c_{L}<c_{H},
$$

and probabilities

$$
\operatorname{Pr}\left(c_{i}=c_{L}\right)=p_{L}, \quad \operatorname{Pr}\left(c_{i}=c_{H}\right)=p_{H}=1-p_{L}, \quad i=1,2 .
$$


Moreover,

$$
\bar{c}:=p_{L} c_{L}+p_{H} c_{H}
$$

is an individual's expected cost of provision. At the beginning of the game, the individuals know neither their own cost of provision nor their rival's cost, but only that this cost can be high or low, and the corresponding probabilities. ${ }^{5}$

In stage 1 of the game, the individuals can find out about their own provision cost: if an individual decides to become informed, he privately observes his provision cost. Information acquisition does not involve any direct cost, and the decisions whether or not to obtain information are made simultaneously and become commonly known at the end of stage 1 .

In stage 2 , the individuals $i=1,2$ simultaneously choose a time of concession $t_{i}$, i.e., individual $i$ plans to provide the public good in $t_{i}$ if individual $j \neq i$ has not volunteered before $t_{i}$. As soon as one individual volunteers, the game ends. However, there is a maximum waiting time $T$ which is exogenously given and common knowledge. Thus, the strategy space is restricted to $t_{i} \in[0, T]$. If both individuals volunteer exactly at the same time, the provision of the public good is allocated with equal probability to the individuals. Waiting involves a direct cost to both individuals, which is assumed to be linear in the waiting time. ${ }^{6}$ Stage 2 is strategically equivalent to the war of attrition or second-price all-pay auction with a cap on bidding.

Denoting by $v$ an individual's utility from the provision of the public good, the

\footnotetext{
${ }^{5}$ The assumption of a discrete distribution determines the structure of the equilibrium strategies in the war of attrition if at least one individual learned his cost. The result on incentives to become informed qualitatively carries over to the case where the individuals' cost is drawn from a continuous distribution. See the discussion in the concluding section.

${ }^{6}$ If the individuals have identical and strictly increasing cost functions $b\left(t_{i}\right)$ for the waiting time $t_{i}$, the analysis can be carried out in a similar way by employing $k_{i}=b\left(t_{i}\right)$ as choice variable.
} 
payoff functions are given by

$$
\pi_{i}\left(t_{i}, t_{j}\right)=\left\{\begin{array}{ll}
v-t_{j}, & t_{i}>t_{j} \\
v-\frac{c_{i}}{2}-t_{i}, & t_{i}=t_{j} \\
v-c_{i}-t_{i}, & t_{i}<t_{j}
\end{array}, i=1,2\right.
$$

For all possible $t_{1}$ and $t_{2}$, the public good is provided, and its value $v$ to the individuals is assumed to be the same for both individuals and independent of the provision time. The idiosyncrasies are captured by the provision cost. The individual who chooses the lower waiting time has to bear the provision cost, and both individuals have to pay the cost of waiting, determined by the minimum of $t_{1}$ and $t_{2}$. If both individuals decide not to concede before $T$, that is $t_{1}=t_{2}=T$, one of them is randomly selected to provide the public good, and their expected payoff in this case is equal to $v-c_{i} / 2-T$.

\section{The volunteering game}

This section analyzes the war of attrition in isolation, fixing the decisions on information acquisition. The equilibrium concept is Bayesian Nash equilibrium. Whenever players are symmetric in the sense that both have (have not) acquired information, the analysis will focus on symmetric equilibria of the war of attrition. ${ }^{7}$

In the war of attrition, the individuals choose their time of concession $t_{i}$, knowing the decisions on information. The time horizon $T$ affects the properties of the equilibrium of the war of attrition for all possible stage 1 decisions. Compared to a provision in $t_{i}<T$, individuals can reduce their expected cost of provision by waiting until $T$ and then possibly being subject to a random selection. This trade-off between lower expected provision cost and higher cost of waiting generates a time interval before $T$ in which, in equilibrium, there is zero probability that an individual volunteers.

\footnotetext{
${ }^{7}$ In the next section, decisions on information acquisition will be considered in a $2 \times 2$ game defined by the payoffs in the war of attrition for the respective information structure.
} 
Lemma 1 Consider the war of attrition for a given information structure. In any equilibrium of the war of attrition, there is zero probability that individual $i$ with cost $c_{i}$ provides the public good in $\left(-\frac{c_{i}}{2}+T, T\right)$.

For a large $T$, it will always be an equilibrium of the volunteering game that an individual $j$ volunteers immediately. In this case, the equilibrium strategy of $i$ is not uniquely determined, and he may choose a concession time $t_{i} \in\left(-c_{i} / 2+T, T\right)$, given that in equilibrium he will not provide the public good. Any $t_{i} \in\left(-c_{i} / 2+T, T\right)$, however, is weakly dominated, and whenever there is positive probability that $j$ waits until $T$, individual $i$ (with cost $c_{i}$ ) strictly prefers $t_{i}=T$ to any $t_{i} \in\left(-c_{i} / 2+T, T\right.$ ). If $T<c_{i} / 2$, we have $-c_{i} / 2+T<0$, and $i$ prefers the random selection in $T$ to a contribution in any $t_{i}<T$. Lemma 1 holds independently of $c_{i}$ being $i$ 's true or expected cost of provision; therefore, it can also be employed if individual $i$ decides not to become informed.

In what follows, we will focus on the case of an intermediate time limit $T$ :

Assumption $1 \frac{c_{L}}{2}<T<\frac{c_{H}}{2}$.

As will become clear in the remainder of this section, Assumption 1 implies that an individual with high cost will find it optimal to wait until $T$, accepting the consequence that he might be randomly chosen to fulfill the task. An individual with low cost will prefer an early concession if the rival waits sufficiently long. ${ }^{8}$

Building on this assumption, we first determine the equilibria of the volunteering game conditional on the decisions in stage 1 , and we then analyze the incentives to become informed in a $2 \times 2$ game defined by the ex ante expected payoffs in the war of attrition. Ex ante expected payoffs are defined as the individuals' expected payoffs given the decisions on information, but before they find out about their provision cost.

\footnotetext{
${ }^{8}$ This assumption ensures the strategic role of the information acquisition because the equilibrium of the volunteering game will crucially depend on the individuals' decisions whether or not to find out about their cost of provision. If $T>c_{H} / 2$, there is always an equilibrium of the war of attrition where one individual concedes immediately, independently of the decisions in stage 1 and the individuals' true provision cost. If $T<c_{L} / 2$, in the unique equilibrium of the war of attrition, both individuals wait until $T$ independently of the stage 1 decisions and their true cost.
} 
In the analysis of the war of attrition, if individual $i$ knows his cost of provision, we will denote by $i_{L}\left(i_{H}\right)$ player $i$ with low (high) cost of provision. Moreover, we will have to allow individuals to randomize their concession time. Consequently, a mixed strategy of an uninformed individual $i \in\{1,2\}$ will be a cumulative distribution function $F_{i}$. Moreover, $q_{i}(t)$ will be the probability that $i$ concedes exactly at $t$, and it will be employed to describe pure strategies. If $i$ acquires information, we denote by $F_{i_{L}}\left(F_{i_{H}}\right)$ the distribution function that corresponds to the mixed strategy $i$ chooses when his cost is low (high). Again, we will use $q_{i_{L}}(t)$ and $q_{i_{H}}(t)$ to describe type-contingent pure strategies in case $i$ acquires information.

Mixed strategies that individuals choose in the different continuation games will exhibit a common structure. For this purpose, we define a function $\Phi$ as

$$
\Phi\left(t ; c, \bar{t}, q^{0}\right)= \begin{cases}1-\left(1-q^{0}\right) e^{-\frac{t}{c}}, & 0 \leq t<\bar{t} \\ 1-\left(1-q^{0}\right) e^{-\frac{\bar{t}}{c}}, & \bar{t} \leq t<T \\ 1, & t \geq T\end{cases}
$$

$\Phi\left(t ; c, \bar{t}, q^{0}\right)$ describes a cumulative distribution function of concession times $t$ with positive mass in the interval $(0, \bar{t})$, no mass in $(\bar{t}, T)$, and possibly a mass point at zero (of size $q^{0}$ ) and/or a mass point at $T$.

No individual knows his cost of provision. If neither of the individuals knows his true provision cost, both choose their waiting time based on their expected cost $\bar{c}$, and the volunteering game is strategically equivalent to the war of attrition with complete information. ${ }^{9}$

Consider individual $i$ and suppose that $j$ waits until $T$ with probability one. If $i$ concedes in $t_{i}<T$, his expected payoff is $v-\bar{c}-t_{i}$. For $t_{i}=T$, he gets a payoff of $v-\bar{c} / 2-T$. Thus, if $T<\bar{c} / 2, t_{i}=T$ is strictly preferred to any $t_{i}<T$, and there is an equilibrium where both wait until $T$ with probability one, which is the unique equilibrium. If, however, $T>\bar{c} / 2$, $i$ 's best response to $t_{j}=T$ is to

\footnotetext{
${ }^{9}$ This holds because individuals are assumed to be risk-neutral and the payoffs are linear in the provision cost. Thus maximizing expected payoffs is equivalent to the maximization based on the expected cost.
} 
concede immediately, and there are two equilibria, each with one individual choosing $q_{i}(0)=1, i=1,2$. In the latter case, there are also equilibria in mixed strategies. ${ }^{10}$ As players are symmetric, we focus on the (unique) symmetric equilibrium.

Lemma 2 (No individual is informed.)

a) If $T \leq \bar{c} / 2$, in the symmetric equilibrium, $q_{1}(T)=q_{2}(T)=1$.

b) If $T>\bar{c} / 2$, in the symmetric equilibrium, individual $i \in\{1,2\}$ randomizes his concession time according to $F_{i}(t)=\Phi\left(t ; \bar{c},-\frac{\bar{c}}{2}+T, 0\right)$.

In the mixed strategy equilibrium (case $T>\bar{c} / 2$ ), for any $t_{j} \in(0,-\bar{c} / 2+T), j$ 's marginal cost of waiting is one, multiplied by the probability $\left(1-F_{i}\left(t_{j}\right)\right)$ that this waiting cost has to be paid. The marginal gain of waiting slightly longer is equal to $\bar{c} F_{i}^{\prime}\left(t_{j}\right)$, i.e. the expected provision cost multiplied by the additional probability that this cost can be saved. Individual $j$ is indifferent between all $t_{j} \in(0,-\bar{c} / 2+T)$ if cost and benefit of increasing $t_{j}$ (i.e. of waiting slightly longer) are equal. This leads to $F_{i}(t)=\Phi\left(t ; \bar{c},-\frac{\bar{c}}{2}+T, 0\right)$. The only difference to the standard war of attrition with complete information is that, due to the time limit, no individual concedes in $(-\bar{c} / 2+T, T)$, but instead both choose a concession in $T$ with strictly positive probability.

In the symmetric equilibrium, no individual concedes immediately with positive probability (that is, $q^{0}=0$ ). There are asymmetric mixed strategy equilibria where one of the individuals places a mass point at $t=0$, i.e. concedes immediately with strictly positive probability. Obviously, there can't be an equilibrium where both individuals have a mass point at zero, because then waiting an infinitesimally small amount of time would, at a negligibly higher expected waiting cost, strictly increase the probability that the rival provides the public good.

The fixed time limit has an important impact on the individuals' equilibrium behavior if $T>\bar{c} / 2$. At the beginning of the game, the individuals are willing to concede, and they play a mixed strategy for a certain time period $(t \in(0,-\bar{c} / 2+T))$.

\footnotetext{
${ }^{10}$ For a detailed analysis see Hendricks et al. (1988).
} 
As the time limit approaches, it becomes less costly to wait until the end, and thus there is a point in time after which the individuals are inactive (for all $t \in$ $(-\bar{c} / 2+T, T))$ because they prefer the random selection at $T$. Finally, they put the remaining probability mass on a concession at $T$.

From Lemma 2, we can compute the individuals' expected payoff in the symmetric equilibrium, which is equal to

$$
E\left(\pi_{i}\right)=\left\{\begin{array}{lll}
v-\bar{c} / 2-T & \text { if } & T \leq \bar{c} / 2 \\
v-\bar{c} & \text { if } & T>\bar{c} / 2
\end{array}, i=1,2 .\right.
$$

One individual knows his cost of provision. Suppose that only individual $j$ has become informed about his provision cost, while $i \neq j$ remained uninformed. $j$ 's strategy is now contingent on his type (denoted by $j_{L}$ or $j_{H}$ ), and $i$ 's optimal strategy is to choose his concession time as if his cost was $\bar{c}$. Recall that we still assume that Assumption 1 holds.

Lemma 3 (One individual is informed.)

a) If $T \leq \bar{c} / 2$, in equilibrium, $q_{i}(T)=q_{j_{H}}(T)=1$, and $q_{j_{L}}(0)=1$. (If $T=\bar{c} / 2$, there is an additional equilibrium where $q_{i}(0)=1$ and $q_{j_{L}}(T)=1$.)

b) If $\bar{c} / 2<T<\bar{c} / 2-\bar{c} \ln p_{H}$,

(i) there is a pure strategy equilibrium where $q_{i}(0)=1$;

(ii) there is a mixed strategy equilibrium where $F_{i}(t)=\Phi\left(t ; c_{L},-\frac{\bar{c}}{2}+T, 0\right)$, $F_{j_{L}}(t)=\frac{1}{p_{L}} \Phi\left(t ; \bar{c},-\frac{\bar{c}}{2}+T, 1-\left(1-p_{L}\right) e^{-\frac{1}{2}+\frac{T}{\bar{c}}}\right)$, and $q_{j_{H}}(T)=1$.

c) If $T \geq \bar{c} / 2-\bar{c} \ln p_{H}$, in equilibrium, $q_{i}(0)=1$.

If $T<\bar{c} / 2$, both $i$ and $j_{H}$ prefer a random selection at $T$ to any concession before $T$, and this makes it optimal for $j_{L}$ to concede immediately. Since there is positive probability that the time limit $T$ is reached, the equilibrium strategies of $i$ and $j_{H}$ are uniquely pinned down.

If $T>\bar{c} / 2$, the structure of the equilibrium reverses, and there is a 'pure strategy equilibrium' where $i$ concedes immediately and both $j_{L}$ and $j_{H}$ wait until $T$. To 
be precise, there is a continuum of payoff-equivalent equilibria where $i$ concedes immediately and $j$ chooses a (sufficiently) high waiting time for each of the two possible provision costs he could have been informed of (sufficiently high to make it optimal for $i$ to concede immediately). Given that Assumption 1 holds, by Lemma 1, $j_{H}$ will never provide the public good with strictly positive probability before $T$. Thus, there is no further pure strategy equilibrium. To see why, suppose that $i$ concedes in $t^{\prime}>0$ with probability one. $j_{L}$ 's best response is either $t_{j_{L}}=0$, or $t_{j_{L}}>t^{\prime}$, and $i$ strictly prefers a concession in $t^{\prime} / 2$ over a concession in $t^{\prime}$ since in both cases this doesn't change his probability of contribution, but strictly reduces the expected waiting cost.

There can, however, be an additional equilibrium which is in mixed strategies. In fact, if $\bar{c} / 2<T<\bar{c} / 2-\bar{c} \ln p_{H}$, there is a 'mixed strategy equilibrium' where $i$ and $j_{L}$ randomize their concession time. By Assumption 1 and Lemma 1, $j_{H}$ will never provide the public good before $T$. Thus, in any equilibrium in mixed strategies, only $i$ and $j_{L}$ contribute before $T$ with strictly positive probability, and the equilibrium strategies exhibit similar properties as in the case of complete information.

Contrary to the case where no individual knows his cost, the mixed strategy equilibrium is uniquely determined by the condition that there is zero probability that any individual concedes in $(-\bar{c} / 2+T, T)$ and that therefore $j_{L}$ concedes before $-\bar{c} / 2+T$ with probability one (see Appendix). This requires that $F_{j_{L}}$ has a mass point at zero, and thus $i$ 's payoff in the mixed strategy equilibrium is strictly higher than $v-\bar{c}$, which is $i$ 's payoff from conceding immediately.

The mixed strategy equilibrium characterized in Lemma 3b(ii) has several interesting properties. Whenever $p_{H}$ and/or $T$ are large, this equilibrium does not exist: as it is likely that $j$ has a high cost and the waiting time until $T$ is costly, waiting becomes too costly for individual $i$; thus $i$ prefers to volunteer immediately. When $T \rightarrow \bar{c} / 2-\bar{c} \ln p_{H}$ (from below), the probability that individual $j_{L}$ concedes immediately converges to zero, and $i$ 's expected payoff converges to $v-\bar{c}$, which is equal to his payoff in the pure strategy equilibrium. On the other hand, when $T \rightarrow \bar{c} / 2$ (from above), the probability that $j_{L}$ concedes immediately converges to one, and the probability that $i$ concedes before $T$ converges to zero. The equilibrium strategies in 
the mixed strategy equilibrium and the individuals' expected payoffs converge to the equilibrium for $T<\bar{c} / 2$. Since individuals are not symmetric in this continuation game and there is no particular reason to focus on one or the other equilibrium, ${ }^{11}$ the analysis of the individuals' incentives to become informed will distinguish which equilibrium is selected in case exactly one individual learned his cost of provision and $T>\bar{c} / 2$.

Given that the pure strategy equilibrium is selected (Lemma $3 b(\mathrm{i})$ ), ex ante expected payoffs are

$$
\begin{aligned}
& E\left(\pi_{i}\right)= \begin{cases}v-p_{H}\left(\frac{\bar{c}}{2}+T\right) & \text { if } T<\frac{\bar{c}}{2} \\
v-\bar{c} & \text { if } T>\frac{\bar{c}}{2}\end{cases} \\
& E\left(\pi_{j}\right)= \begin{cases}v-p_{L} c_{L}-p_{H}\left(\frac{c_{H}}{2}+T\right) & \text { if } T<\frac{\bar{c}}{2} \\
v & \text { if } T>\frac{\bar{c}}{2}\end{cases}
\end{aligned}
$$

In case the mixed strategy equilibrium is selected (Lemma 3b(ii)), ex ante expected payoffs equal

$$
\begin{aligned}
& E\left(\pi_{i}\right)= \begin{cases}v-p_{H}\left(\frac{\bar{c}}{2}+T\right) & \text { if } T<\frac{\bar{c}}{2} \\
v-p_{H} e^{-\frac{1}{2}+\frac{T}{\bar{c}}} \bar{c} & \text { if } \frac{\bar{c}}{2}<T<\frac{\bar{c}}{2}-\bar{c} \ln p_{H} \\
v-\bar{c} & \text { if } T \geq \frac{\bar{c}}{2}-\bar{c} \ln p_{H}\end{cases} \\
& E\left(\pi_{j}\right)= \begin{cases}v-p_{L} c_{L}-p_{H}\left(\frac{c_{H}}{2}+T\right) & \text { if } T<\frac{\bar{c}}{2} \\
v-c_{L}-\frac{p_{H}}{2} e^{\frac{\bar{c}-2 T}{2 c_{L}}}\left(c_{H}+\bar{c}-2 c_{L}\right) & \text { if } \frac{\bar{c}}{2}<<<\frac{\bar{c}}{2}-\bar{c} \ln p_{H} \\
v & \text { if } T \geq \frac{\bar{c}}{2}-\bar{c} \ln p_{H}\end{cases}
\end{aligned}
$$

For $T<\bar{c} / 2, j_{L}$ concedes immediately; therefore, the expected payoff of the uninformed individual $i$ increases with the probability that $j$ has a low contribution cost. Note that in this case $E\left(\pi_{i}\right)>E\left(\pi_{j}\right)$, i.e. the individual who does not know his cost of provision has a higher expected payoff than the informed individual. For a large $T$, however, the uninformed individual may concede immediately and gets a lower expected payoff.

\footnotetext{
${ }^{11}$ In particular, the two equilibria cannot be Pareto-ranked.
} 
Both individuals know their cost of provision. Suppose that both individuals have decided to acquire information about their provision cost. By Lemma 1 together with Assumption 1, there can't be an equilibrium where a type of $i$ with high cost, $i_{H}$, provides the public good in $t_{i_{H}}<T$ with strictly positive probability. If $i_{H}$ chooses a time of concession $t_{i_{H}}<T$ with strictly positive probability, then $j_{H}$ must concede before $t_{i_{H}}$ with probability one, contradicting Lemma 1. Therefore, in any equilibrium, $q_{i_{H}}(T)=q_{j_{H}}(T)=1$.

It remains to characterize the individuals' equilibrium strategies for a low provision cost. As before, denote by $i_{L}$ an individual $i$ with low cost. There can't be an equilibrium where $i_{L}$ chooses a pure strategy. In particular, there can't be an equilibrium where an individual with low cost volunteers immediately. To see why, suppose that $i_{L}$ chooses $t=0$ with probability one. $j_{L}$ 's best response is to concede in $t^{\prime}=\varepsilon, \varepsilon$ infinitesimally small, knowing that $i_{H}$ will wait until $T$. But then, $i_{L}$ is strictly better off by choosing $t^{\prime \prime}=2 \varepsilon$.

Hence, individuals randomize their waiting time if they have a low provision cost. By Lemma 1, there must be zero probability that an individual volunteers in the interval $\left(-c_{L} / 2+T, T\right)$, and at most one individual can have a mass point at zero. As it is a typical feature of the war of attrition, there may be a continuum of equilibria which differ in the size of the mass point at zero. Since the individuals are symmetric ex ante, we focus on the symmetric equilibrium.

Lemma 4 (Both individuals are informed.)

In the symmetric equilibrium, $q_{i_{H}}(T)=1$ and $F_{i_{L}}(t)=\frac{1}{p_{L}} \Phi\left(t ; c_{L}, \bar{t}, 0\right)$ where $\bar{t}=$ $\min \left\{-\frac{c_{L}}{2}+T,-c_{L} \ln p_{H}\right\}, i=1,2$.

If the probability $p_{H}$ that the other individual has a high cost is large, it is more attractive for an individual with low cost to volunteer early. For sufficiently high $p_{H}$, $i_{L}$ and $j_{L}$ concede before $T$ with probability one. This holds if $-\frac{c_{L}}{2}+T \geq-c_{L} \ln p_{H}$ or

$$
T \geq \frac{c_{L}}{2}-c_{L} \ln p_{H}
$$


Otherwise, the low types put strictly positive probability on a concession in $T$, as waiting until $T$ is less costly. Again, up to a point in time $\bar{t}$, there is a positive probability that an individual concedes in case he has a low cost, and there is a time period just before $T$ where both individuals are inactive, since they prefer to wait until $T$ if the cost of the additional waiting time is sufficiently low. Ex ante expected payoffs are

$$
E\left(\pi_{i}\right)= \begin{cases}v-c_{L}-\frac{p_{H}}{2}\left(c_{H}-c_{L}\right) e^{\frac{1}{2}-\frac{T}{c_{L}}} & \text { if } T<\frac{c_{L}}{2}-c_{L} \ln p_{H} \\ v-c_{L}-p_{H}^{2}\left(T+\frac{c_{H}}{2}-c_{L}\left(1-\ln p_{H}\right)\right) & \text { if } T \geq \frac{c_{L}}{2}-c_{L} \ln p_{H}\end{cases}
$$

for $i=1,2$.

\section{The value of becoming informed}

This section considers the decisions on information acquisition in a $2 \times 2$ game defined by the payoffs in the war of attrition that have been determined in the previous section. ${ }^{12}$ Let $\sigma_{i} \in\{N, I\}$ be an individual $i$ 's decision on information where $I$ refers to information acquisition and $N$ to a decision not to learn one's own provision cost. Moreover, denote by $E\left(\pi_{i}^{\left(\sigma_{i}, \sigma_{j}\right)}\right)$ individual $i$ 's ex ante expected payoff in the war of attrition given the decisions $\left(\sigma_{i}, \sigma_{j}\right)$. In case $(I, I)$, for instance, both individuals have learned their cost of provision, whereas case $(N, I)$ refers to a situation where exactly one individual has decided to learn his cost. Given $\sigma_{j}, i$ 's value of information can be defined as

$$
V_{i}^{\sigma_{j}}=E\left(\pi_{i}^{\left(I, \sigma_{j}\right)}\right)-E\left(\pi_{i}^{\left(N, \sigma_{j}\right)}\right)
$$

\footnotetext{
${ }^{12}$ This approach is employed to simplify the exposition, and it shows that in the equilibrium of the $2 \times 2$ game, one player may remain uninformed. The equilibria of the reduced game can also be supported as Perfect Bayesian equilibria in the analysis of the two-stage game, assuming beliefs about the rival's type that do not change with the information acquisition decision (players have no private information when deciding whether to acquire information).
} 
For the analysis of the optimal decision on information acquisition, we have to distinguish whether or not $T>\bar{c} / 2$. This distinction does not influence the equilibrium of the war of attrition in case both individuals know their provision cost, but it is crucial for the nature of the equilibrium if at least one individual does not know his cost of provision. ${ }^{13}$

Lemma 5 Suppose that Assumption 1 holds.

(i) $V_{i}^{\sigma_{j}=N}$ is strictly positive for all $T$.

(ii) $V_{i}^{\sigma_{j}=I}$ is strictly negative if $T$ is sufficiently small and strictly increasing in $T$ for $T \in\left(c_{L} / 2, \bar{c} / 2\right)$.

(iii) Suppose in case $(N, I)$ the pure strategy equilibrium is selected. Then $V_{i}^{\sigma_{j}=I}$ is strictly positive for all $T>\bar{c} / 2$.

(iv) Suppose in case $(N, I)$ the mixed strategy equilibrium is selected. Then $V_{i}^{\sigma_{j}=I}$ is continuous and strictly increasing in $T$ for $T \in\left(c_{L} / 2, \bar{c} / 2-\bar{c} \ln p_{H}\right)$.

Provided that the rival does not learn his cost of provision $\left(\sigma_{j}=N\right)$, learning one's own cost always increases one's expected payoff as the value of information is positive (Lemma 5 part (i)). If instead the rival decides to learn his cost and $T$ is small, this result is reversed. However, as long as $T<\bar{c} / 2$, an increasing time limit makes waiting more costly in case the rival has a high provision cost, which increases one's own value of information (part (ii)). If $T>\bar{c} / 2$, the value of information depends on which equilibrium is selected in case $(N, I)$. For the pure strategy equilibrium, $i$ 's value of information given that $j$ learns his cost of provision, $V_{i}^{I}$, exhibits a discontinuity at $T=\bar{c} / 2$ and is strictly positive for all $T>\bar{c} / 2$ (part (iii)). For the mixed strategy equilibrium, however, $V_{i}^{I}$ is continuous at $T=\bar{c} / 2$. This continuity in $T$ makes the analysis for the selected equilibrium more appealing. Yet the following proposition holds independently of which equilibrium is selected in case only one individual decides to learn his provision cost. ${ }^{14}$

\footnotetext{
${ }^{13}$ We still assume that Assumption 1 holds. If $T<c_{L} / 2$, then decisions on information are irrelevant, since both individuals never concede before $T$. If $T>c_{H} / 2$, the war of attrition always has equilibria where one of the individuals concedes immediately, independent of the decisions on information.

${ }^{14}$ Due to the possible multiplicity of equilibria of the war of attrition in case of $T>\bar{c} / 2$, departing from the analysis of the reduced form game makes the equilibrium analysis more complex in
} 
Proposition 1 Consider the game of information acquisition and suppose that Assumption 1 holds. There exists a threshold $\tilde{T}>c_{L} / 2$ such that

(i) if $T<\tilde{T}$, there are two asymmetric equilibria where exactly one individual acquires information and one symmetric equilibrium where both individuals randomize their information decision;

(ii) if $T>\tilde{T}$, it is strictly dominant to acquire information.

If both individuals remained uninformed, this would cause a high inefficiency in the volunteering game and lead to the lowest expected payoffs. Therefore, it is beneficial for at least one individual to find out about his provision cost even if information acquisition leads to a higher ex ante probability of being the one who concedes first. As a consequence, there is never an equilibrium where both individuals decide not to learn their cost of provision. If, however, $T$ is sufficiently small and only individual $j$ acquires information, then $j$ concedes immediately with high probability, and $i$ prefers to remain uninformed. Being uninformed constitutes a strategic advantage in the volunteering game, being a commitment not to volunteer too early. This, in turn, induces the rival to concede immediately, which outweighs $i$ 's waiting cost in case $j$ has a high provision cost. For a higher $T$, this waiting cost increases, and, in the case of the mixed strategy equilibrium in $(N, I)$, the probability that $j$ concedes immediately decreases. There exists a threshold $\tilde{T}$ such that, for $T>\tilde{T}, i$ is better off if he finds out about his provision cost as well. If the value of information $V_{i}^{I}$ is negative for all $\left(c_{L} / 2, \bar{c} / 2\right)$, the location of $\tilde{T}$ depends on which equilibrium is selected in case $(N, I)$. In both cases, the threshold $\tilde{T}$ is uniquely determined such that $V_{i}^{I}$ is negative for all $T<\tilde{T}$ and positive for all $T>\tilde{T}$.

Corollary 1 (i) If in case $(N, I)$ the pure strategy equilibrium is selected, $\tilde{T} \leq \bar{c} / 2$. (ii) If in case $(N, I)$ the mixed strategy equilibrium is selected and $p_{H}$ is small, $\tilde{T}$ is strictly larger than $\bar{c} / 2$. Then, there may be no equilibrium where both individuals acquire information with probability one for all $T$ fulfilling Assumption 1.

this case. Then, players can condition their strategies in the war of attrition on the information acquisition. As in our analysis for the pure strategy equilibrium in case $(N, I)$, this can support information acquisition of both players in equilibrium if $T>\bar{c} / 2$. 
If $T>\bar{c} / 2$ and, in case $(N, I)$, the pure strategy equilibrium is selected, learning the own provision cost is strictly dominant, and thus the threshold $\tilde{T}$ is (weakly) smaller than $\bar{c} / 2 \cdot{ }^{15}$ However, if we focus on the mixed strategy equilibrium, $\tilde{T}>\bar{c} / 2$ for a small $p_{H}$, and the value of information $V_{i}^{I}$ can even be negative for all $T \in$ $\left(c_{L} / 2, c_{H} / 2\right)$. Thus, the strategic value of remaining uninformed is not only present in the case where an uninformed individual $i$ has a dominant strategy not to concede before $T$ (as in Lemma 3a), but also when the individuals randomize their concession time (as in Lemma 3b(ii)). The sufficiently high probability that the rival has a low cost and volunteers immediately with positive probability makes it optimal for $i$ to disregard information that is available without cost. This strategic value disappears only if the probability of having a high contribution cost, $p_{H}$, is large, because, from the point of view of the rival, an early concession of the individual who knows his provision cost is less likely.

Example Consider the following example where $c_{L}=2$, and $c_{H}=10$. Assumption 1 requires that $1<T<5$.

(a) Suppose that $p_{H}=0.75$. If $T \rightarrow \bar{c} / 2=4$ from below, the value of information $V_{i}^{I}$ is positive. Hence, the critical threshold $\tilde{T}<\bar{c} / 2$. Setting $V_{i}^{I}(T)=0$ yields $\tilde{T}=1.94$. Thus, for all $T<1.94$, only one individual learns his cost of provision, and for all $T>1.94$, both individuals learn their cost of provision.

(b) Now suppose that $p_{H}=0.5 . V_{i}^{I}$ is negative if $T$ approaches $\bar{c} / 2=3$ (from below). Hence, if in case $(N, I)$ the pure strategy equilibrium is selected, $\tilde{T}=\bar{c} / 2=3$, and if the mixed strategy equilibrium is selected, $\tilde{T}>\bar{c} / 2$. In the latter case, $\tilde{T}=3.56$.

(c) If $p_{H}=0.25$, again $V_{i}^{I}$ is negative if $T$ approaches $\bar{c} / 2=2$, and $\tilde{T}=\bar{c} / 2$ if in case $(N, I)$ the pure strategy equilibrium is selected. If the mixed strategy equilibrium is selected, $V_{i}^{I}$ is negative for all $T$ satisfying Assumption 1, and thus there is no equilibrium where both individuals find out about their cost of provision with probability one.

\footnotetext{
${ }^{15}$ Concretely, if $V_{i}^{\sigma_{j}=I}$ is negative for all $T<\bar{c} / 2, \tilde{T}=\bar{c} / 2$. Otherwise, $\tilde{T}$ is defined as the solution to $V_{i}^{\sigma_{j}=I}(\tilde{T})=0$.
} 


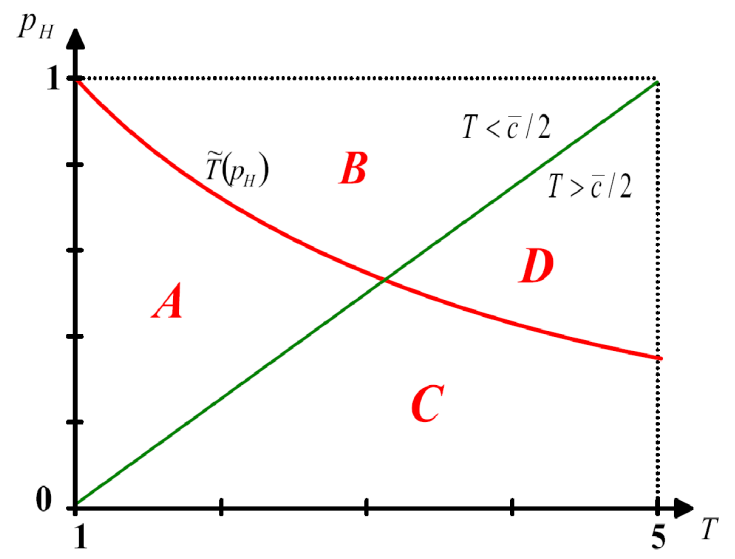

Figure 1: Equilibrium information acquisition (for $c_{L}=2, c_{H}=10$ ).

Figure 1 shows the equilibrium outcome for different combinations of $T$ and $p_{H}$. The 45-degree line describes the condition $T=\bar{c} / 2$. In the areas $B$ and $D$, finding out about the own cost of provision is strictly dominant; in area $A$, the individuals prefer to remain uninformed if the rival acquires information, and in equilibrium only one individual learns his cost (or both individuals randomize their information acquisition decision). In area $C$, the outcome depends on which equilibrium is selected in case $(N, I)$. Here, $T>\bar{c} / 2$, and for the pure strategy equilibrium, information acquisition is strictly dominant. For the mixed strategy equilibrium, however, only one individual acquires information.

A designer's perspective. There are several dimensions along which efficiency can be defined. On the one hand, a designer could be interested in the individual with the lowest cost (highest ability) providing the public good. On the other hand, the designer might want to minimize the expected waiting time. ${ }^{16}$ To capture these different dimensions, consider the following objective function

$$
W=2 v-\lambda_{1} E\left(\min \left\{t_{1}, t_{2}\right\}\right)-\lambda_{2} E\left(k\left(t_{1}, t_{2}\right)\right)
$$

\footnotetext{
${ }^{16}$ In a framework of a contest, a designer may want to induce long times of fighting, i.e. high waiting times.
} 
where

$$
k\left(t_{1}, t_{2}\right)=\left\{\begin{array}{ccc}
c_{1} & \text { if } t_{1}<t_{2} \\
\left(c_{1}+c_{2}\right) / 2 & \text { if } t_{1}=t_{2} \\
c_{2} & \text { if } t_{1}>t_{2}
\end{array}\right.
$$

is the (expected) cost of providing the public good and $\lambda_{1}$ and $\lambda_{2}$ are the weights given to the expected waiting time and the expected provision cost. We assume that the designer does not know the individuals' cost of provision and cannot change the structure of the game.

Suppose first that $\lambda_{1}=0$ and $\lambda_{2}>0$, that is, maximizing $W$ is equivalent to minimizing the expected cost of provision, $E\left(k\left(t_{1}, t_{2}\right)\right)$. Here, $W$ is highest if both individuals acquire information (case $(I, I)$ ) and an individual with low cost volunteers with probability one before the time limit is reached. This implies that $T>c_{L} / 2-c_{L} \ln p_{H}$ (by Lemma 4 ) and $T>\tilde{T}$ (by Proposition 1). In this case, information acquisition is efficient.

Remark 1 If the designer wants to minimize the expected cost of provision, a sufficiently high time limit ensures both efficient information acquisition and efficient provision of the public good.

Another objective could be to focus on the expected waiting time. Let $\lambda_{2}=0$. Obviously, if $\lambda_{1}>0$, the time horizon should be as short as possible, and $W$ is maximized for $T=0$. In this case, the decisions on information become irrelevant. ${ }^{17}$

If the designer takes into account both the expected cost of provision and the expected waiting cost, a benevolent designer may want to maximize the individuals' expected payoffs, which is equivalent to $\lambda_{1}=2$ and $\lambda_{2}=1$. Then, $T=0$ need not be

\footnotetext{
${ }^{17}$ If $T>\bar{c} / 2$ and in case $(N, I)$ the pure strategy is selected, $W$ would also be maximized if exactly one individual acquires information. This, however, does not occur in equilibrium if the individuals decide on information acquisition, but only if information acquisition is forbidden for one individual. In this sense, there can be too much information acquisition in equilibrium if $\lambda_{1}>0$ and $\lambda_{2}=0$. If instead $\lambda_{1}<0$ and the designer wants to maximize the expected waiting time, the waiting times are highest if $T>\bar{c} / 2$ and none of the individuals acquires information. Thus, it would be optimal to prohibit information acquisition.
} 
optimal: if $T$ is only slightly larger than $c_{L} / 2$, one individual acquires information, and he concedes immediately in case he has a low cost. The gain from the decrease in the expected provision cost (due to information acquisition) outweighs the higher waiting cost if $p_{H}$ is sufficiently small and/or $c_{H}$ is large, and it can be optimal to choose an intermediate time limit such that individuals have an incentive to acquire information and to choose an early concession if they have a low provision cost. Similarly, it can be desirable that both individuals acquire information. In the latter case (case $(I, I))$, the sum of expected payoffs is highest if $T=c_{L} / 2-c_{L} \ln p_{H}$ such that individuals with low cost concede before $T$ with probability one. Higher $T$ do not change the efficiency of the provision (captured by $k\left(t_{1}, t_{2}\right)$ ), but increase the waiting cost given that both individuals have a high cost. In general, the optimal choice of $T$ depends on the balancing of expected waiting time and cost of provision and on the probability of facing individuals with a high cost of provision.

Remark 2 If the designer wants to maximize the sum of expected payoffs, the tradeoff between efficiency of the provision and cost of waiting makes an intermediate time limit optimal whenever $p_{H}$ is sufficiently small and/or $c_{H}$ is large.

\section{Extensions}

Sequential decisions on information. Whenever there is an incentive to remain uninformed, this can cause a coordination problem. When individuals randomize their information acquisition decision, they may acquire too much or too little information from their own point of view. Considering sequential choices on information can mitigate this coordination problem, and it will reflect, for instance, situations where individuals can, one after the other, ask questions about a task that has to be performed.

Suppose that decisions on information take place sequentially: individual 1 decides first, and individual 2 moves second. ${ }^{18}$ As stated in Proposition 1, information

\footnotetext{
${ }^{18}$ We do not discuss the question of endogenous timing of information acquisition decisions.
} 
acquisition is strictly dominant for $T>\tilde{T}$, and both individuals will acquire information. For $T<\tilde{T}$, however, if individual 1 acquires information, 2 will remain uninformed, and vice-versa.

Proposition 2 Suppose that decisions on information take place sequentially and Assumption 1 holds. If the time horizon is sufficiently small, the first mover will decide to remain uninformed, and the second mover will acquire information.

Whenever $T<\bar{c} / 2$ and exactly one individual has acquired information, the payoff of the uninformed individual is higher than the payoff of the informed individual. Thus, individuals prefer to be the uninformed player. If $T>\bar{c} / 2$, we have to distinguish which equilibrium is selected in case $(N, I)$. For the mixed strategy equilibrium, a strategic incentive to remain uninformed exists, and an increasing time horizon $T$ makes it less attractive to remain uninformed. There is, however, a range of parameters $T$ where the strategic advantage from being uninformed is sufficiently high such that a first mover would choose to remain uninformed.

Information about a common value. In the previous section, we have identified a strategic value of ignorance in situations where information about a private value can be obtained. If the information is about some component which is common to all individuals, a similar strategic incentive is present. Consider the extreme case of a pure common value and suppose that the individuals' costs of provision are perfectly correlated. Thus, if an individual has acquired information, he knows not only his own type, but also his rival's type.

In the war of attrition, if no individual has acquired information, the analysis does not change. Moreover, if both individuals have acquired information, they randomize their concession time if they both have a low cost, and they wait until $T$ if they both have a high cost.

If exactly one individual knows the cost of provision and $T<\bar{c} / 2$, the equilibrium of the war of attrition is similar to the one characterized in Lemma 3a. Here, if the informed individual $j$ does not concede immediately, the uninformed individual $i$ 
knows that his cost is high and will find it optimal to wait until $T{ }^{19}$

If $T>\bar{c} / 2$, the mixed strategy equilibrium of Lemma $3 b(i i)$ does not exist. The intuition is as follows. If there were such an equilibrium, the uninformed individual $i$ would update his beliefs about his cost following the action of this rival, and if the game reaches a point in time $-\frac{\bar{c}}{2}+T-\delta, \delta$ positive but small, $i$ would know almost with certainty that his cost is high. But then, $i$ would not concede in $\left(-\frac{\bar{c}}{2}+T-\delta,-\frac{\bar{c}}{2}+T\right)$, but instead wait until $T$.

For $T \geq \bar{c} / 2$, there is an equilibrium where the uninformed individual concedes immediately $\left(q_{i}(0)=1\right)$. Moreover, contrary to the case of private values, there is an equilibrium where $q_{j_{L}}(0)=1$ and $q_{j_{H}}(T)=q_{i}(T)=1$. Here, $i$ knows that his cost is high if there is no immediate concession of $j$ and thus finds it optimal to wait until $T$. In turn, $j$ cannot profitably deviate given that $q_{i}(T)=1$.

As in the private values case, we consider the $2 \times 2$ game of information acquisition defined by the payoffs in the war of attrition.

Proposition 3 Consider the game of information acquisition with common values and suppose that Assumption 1 holds.

(i) If $T<\bar{c} / 2$, only one individual acquires information in equilibrium;

(ii) if $T \geq \bar{c} / 2$, dependent on the equilibrium selection in the war of attrition (case $(N, I))$, only one individual or both individuals acquire information in equilibrium.

In case of $T \geq \bar{c} / 2$, the war of attrition in case $(N, I)$ has two diametrically opposed equilibria, and decisions on information crucially depend on which of the equilibria is played. For a small $T$, however, as in the case of private values, one individual strategically chooses to remain uninformed of the cost of provision, and in turn the informed individual concedes immediately if the (common) cost is low. ${ }^{20}$

\footnotetext{
${ }^{19}$ There is no further pure strategy equilibrium because, even if $q_{j_{L}}(T)=q_{j_{H}}(T)=1$, $i$ 's best response is $q_{i}(T)=1$. Moreover, there is no mixed strategy equilibrium: intuitively, if $j_{L}$ randomized and $i$ provided the good at some $t>0, i$ would know that his expected cost would be higher than $\bar{c}$ (it becomes more likely that the cost is high); thus, $i$ prefers to wait until $T$.

${ }^{20}$ As in Proposition 1, there are two asymmetric equilibria where exactly one individual acquires information and one symmetric equilibrium where both individuals randomize their information acquisition decision.
} 
Lost opportunity of provision at $T$. The provision of many public goods is allocated on a voluntary basis, but it is compulsory in the sense that one individual has to contribute. In companies, for instance, a team leader may select one individual if no one volunteers. Other public goods can only be provided within a certain period, after which the opportunity of provision disappears.

Instead of assuming that at $T$ one individual is randomly selected, suppose that the investment opportunity disappears if no individual has conceded before $T$. In this case, the incentive to wait until $T$ in the war of attrition is weakened; the analysis, however, qualitatively carries over from the previous section if we modify Assumption 1 on the time limit such that individuals with a high cost do not want to provide the public good and individuals with a low cost prefer to concede. This requires the time limit to be such that:

Assumption 1' $0<-\left(v-c_{L}\right)<T<-\left(v-c_{H}\right)$.

Hence, high types have a (weakly) dominant strategy to wait until T. Moreover, if $T$ is sufficiently small $(T<-(v-\bar{c}))$ and only individual $j$ knows his contribution cost, the uninformed individual $i$ waits until $T$ (as in Lemma 3a). For a larger $T$, there is an equilibrium where $i$ and $j_{L}$ randomize on some interval $[0, \bar{t}] \cup\{T\}$ (similar to Lemma $3 \mathrm{~b}(\mathrm{ii}))$ and $j_{L}$ has a mass point at zero.

Proposition 4 Consider the game of information acquisition and suppose that at $T$ the opportunity of provision disappears. If Assumption 1' holds and T is sufficiently small, only one individual acquires information in equilibrium.

We do not provide a complete analysis of equilibria of the war of attrition ${ }^{21}$ and incentives to acquire information, but we show that, whenever $T$ is small, there is a strategic value of ignorance: as in the previous section, remaining uninformed can be used as a commitment not to concede if an individual's expected cost of provision is sufficiently high in relation to the payoff from waiting until $T$.

\footnotetext{
${ }^{21}$ A detailed analysis of equilibria of the war of attrition would build on Theorems 1-3 in Hendricks et al. (1988).
} 


\section{Conclusion}

The private provision of a discrete public good is likely to end up in a war of attrition: individuals prefer to wait until someone else volunteers and provides the public good. But they may not be able to wait for an infinite amount of time. This can be due to time constraints or to a finite time horizon imposed by a third party. In many applications, such as allocating tasks in firms or communities, time limits are a typical feature of the volunteering game.

In this paper, we analyzed incentives to obtain information ahead of a war of attrition. The information that is available to the individuals has an important impact on the equilibrium outcome of the volunteering game. This suggests that individuals have an incentive to use information acquisition strategically when they anticipate the private provision game. We assumed that initially the individuals do not know exactly their own cost of provision of the public good, but that they can find out about this cost prior to the volunteering game. Indeed, there can be an incentive for one individual not to become informed of his cost of provision even if the information is available without cost. For a sufficiently short time horizon, being uninformed induces an informed individual to volunteer immediately in case he has a low cost of provision, whereas not knowing the own cost of provision constitutes a commitment to delay the own concession. For a sufficiently long time horizon, however, finding out about the own cost is a strictly dominant strategy. Since the time horizon has a crucial impact on information acquisition as well as on the equilibrium outcome of the volunteering game, it may be used as an instrument to influence the efficiency of the public good provision.

Our model assumed that the individuals' costs of provision follow a two-point probability distribution. For continuous distribution functions, similar results can be obtained. The equilibrium properties change in the sense that an individual with private information about his cost of provision chooses his concession time as an increasing function of his provision cost. In the case where exactly one individual has learned his cost, we get a similar result for a small time limit $T$ : the informed individual volunteers immediately if he has a low cost of provision, which creates 
an incentive for the rival to remain uninformed of his own cost. For intermediate values of $T$, a mixed strategy equilibrium exists that exhibits similar properties to the one characterized in Lemma 3b(ii). The value of information is then determined by the shape of the probability distribution of the provision cost. The resulting effects are qualitatively the same, but can be most clearly demonstrated by using a two-point distribution and varying the probabilities that the cost of provision is high and low, respectively. The key assumption remains that with positive probability individuals face a rival who prefers to wait until the time limit is reached. In this sense, our approach is similar to Fudenberg and Tirole (1986), who assume that there is a positive probability that the rival never concedes. For the incentives to find out about the cost of provision, the time limit is of additional strategic importance.

\section{A Appendix}

\section{A.1 Proof of Lemma 1}

Denote by $\Psi_{j}$ the distribution of $j$ 's waiting times, from the point of view of $i$, that is, $\Psi_{j}(t)=a$ means that, from the point of view of $i, j$ concedes before $t$ with probability $a .{ }^{22}$ Consider a concession of $i$ in $t_{i} \in\left(-c_{i} / 2+T, T\right)$ and suppose that there is a strictly positive probability that $i$ provides the good in $t_{i}$, i.e. $\Psi_{j}\left(t_{i}\right)<1$. If $\Psi_{j}$ exhibits a discontinuity at $t_{i}$, then there is an $\varepsilon>0$ such that $i$ is strictly better off by conceding in $t_{i}+\varepsilon$ instead of in $t_{i}$, because this would strictly decrease the expected contribution cost at only an infinitesimally higher expected waiting cost.

\footnotetext{
${ }^{22}$ Note that $\Psi_{j}$ captures both uncertainty of $i$ over $j$ 's contribution cost and possible randomization of $j$.
} 
Otherwise, $i$ 's expected payoff from a concession in $t_{i}$ is

$$
\begin{aligned}
& \int_{0}^{t_{i}}(v-t) d \Psi_{j}(t)+\left(1-\Psi_{j}\left(t_{i}\right)\right)\left(v-t_{i}-c_{i}\right) \\
= & \int_{0}^{T}(v-t) d \Psi_{j}(t)-\int_{t_{i}}^{T}(v-t) d \Psi_{j}(t) \\
& +\left(1-\Psi_{j}(T)\right)\left(v-t_{i}-c_{i}\right)+\left(\Psi_{j}(T)-\Psi_{j}\left(t_{i}\right)\right)\left(v-t_{i}-c_{i}\right) \\
= & \int_{0}^{T}(v-t) d \Psi_{j}(t)+\left(1-\Psi_{j}(T)\right)\left(v-t_{i}-c_{i}\right) \\
& -\int_{t_{i}}^{T}\left(t_{i}+c_{i}-t\right) d \Psi_{j}(t) .
\end{aligned}
$$

$\Psi_{j}\left(t_{i}\right)<1$ implies that $\Psi_{j}(T)-\Psi_{j}\left(t_{i}\right)>0$ or/and $1-\Psi_{j}(T)>0$. Therefore, for all $t_{i} \in\left(-c_{i} / 2+T, T\right),(9)$ is strictly smaller than

$$
\int_{0}^{T}(v-t) d \Psi_{j}(t)+\left(1-\Psi_{j}(T)\right)\left(v-c_{i} / 2-T\right)
$$

which is $i$ 's expected payoff for $t_{i}=T$.

\section{A.2 Proof of Lemma 2}

(i) As argued in the main text, the best response to $t_{j}=T$ is $t_{i}=T$, and $q_{i}(T)=$ $q_{j}(T)=1$ is an equilibrium. Moreover, since $-c_{i} / 2+T<0$, Lemma 1 rules out any further equilibrium because any individual who contributes with strictly positive probability in $t^{\prime} \in[0, T)$ would strictly prefer a concession in $T$ to a concession in $t^{\prime}$. (ii) The structure of the equilibrium strategies follows from Hendricks et al. (1988) and the analysis in the main text. We only show that the strategies constitute an

equilibrium. Suppose that $j$ randomizes according to $F_{j}(t)=\Phi\left(t ; \bar{c},-\frac{\bar{c}}{2}+T, 0\right)$, where $\Phi$ is defined in (2). Then, by Lemma $1, i$ strictly prefers $t_{i}=T$ to any $t_{i} \in(-\bar{c} / 2+T, T)$. For $t_{i} \in[0,-\bar{c} / 2+T], i$ 's payoff is

$$
\int_{0}^{t_{i}}(v-x) \frac{1}{\bar{c}} \exp \left(-\frac{x}{\bar{c}}\right) d x+\exp \left(-\frac{t_{i}}{\bar{c}}\right)\left(v-t_{i}-\bar{c}\right)=v-\bar{c}
$$


and if $t_{i}=T, i$ gets

$$
\int_{0}^{-\frac{\bar{c}}{2}+T}(v-x) \frac{1}{\bar{c}} \exp \left(-\frac{x}{\bar{c}}\right) d x+\exp \left(\frac{1}{2}-\frac{T}{\bar{c}}\right)\left(v-T-\frac{\bar{c}}{2}\right)=v-\bar{c} .
$$

Thus $i$ is indifferent between all $t_{i} \in(0,-\bar{c} / 2+T] \cup\{T\}$, and $F_{i}$ and $F_{j}$ are mutually best responses.

\section{A.3 Proof of Lemma 3}

Parts a), b(i) and c) follow directly from the analysis in the main text. It remains to prove part $\mathrm{b}$ (ii). If there is an equilibrium in mixed strategies, the equilibrium strategies must exhibit similar properties as in the case of complete information. In particular, for waiting times $t_{i}$ and $t_{j_{L}}$ in the support of the mixed strategies, it has to hold that

$$
\begin{aligned}
& F_{i}\left(t_{i}\right)=1-\left(1-b_{i}\right) \exp \left(-\frac{t_{i}}{c_{L}}\right), \\
& F_{j_{L}}\left(t_{j_{L}}\right)=\frac{1}{p_{L}}\left[1-\left(1-p_{L} b_{j_{L}}\right) \exp \left(-\frac{t_{j_{L}}}{\bar{c}}\right)\right],
\end{aligned}
$$

where the constants $b_{i}$ and $b_{j_{L}}$ correspond to the mass points at zero, $F_{i}(0)$ and $F_{j_{L}}(0)$, and remain to be determined. The factor $1 / p_{L}$ in $F_{j_{L}}$ takes into account the probability $p_{L}$ that $i$ faces a rival with cost $c_{L}$. It has to hold that $0 \leq b_{i}, b_{j_{L}}<1$, and $\min \left(b_{i}, b_{j_{L}}\right)=0$ : if $i\left(j_{L}\right)$ concedes immediately with strictly positive probability, $j_{L}$ $(i)$ strictly prefers a concession in $\varepsilon>0, \varepsilon$ infinitesimally small, to a concession in 0 .

Assumption 1 implies that no $t_{j_{H}}<T$ with $F_{i}\left(t_{j_{H}}\right)<1$ can be part of $j_{H}$ 's equilibrium strategy: $j_{H}$ won't choose any $t_{j_{H}}<T$ in the support of $F_{i}$. In turn, for any $t_{i}<T$, we must have $F_{j_{H}}\left(t_{i}\right)=0$, and thus $i$ strictly prefers $t_{i}=T$ to all $t_{i} \in(-\bar{c} / 2+T, T)$. Moreover, $F_{i}$ must be continuous on $(0, T)$. To see why, suppose that $i$ concedes in $t_{i} \in(0,-\bar{c} / 2+T]$ with strictly positive probability. Then, there are $\delta>0, \varepsilon>0$ such that $j_{L}$ strictly prefers $t_{j}=t_{i}+\varepsilon$ to any $t_{j} \in\left(t_{i}-\delta, t_{i}\right)$, hence $i$ is strictly better off by choosing $t_{i}-\delta / 2$ instead of $t_{i}$. Therefore, possible mass 
points of $F_{i}$ are restricted to $t_{i}=0$ and $t_{i}=T$.

We proceed in two steps: first we show that the mass points at zero are uniquely determined, and second we prove that (10) and (11) constitute an equilibrium.

Step 1: From (10), it follows that $b_{i}<1$ implies $F_{i}\left(t_{i}\right)<1$ for all $t_{i}<T$ : whenever $i$ chooses a mixed strategy, there is strictly positive probability that $t_{i}=T$. In particular, we have $F_{i}(-\bar{c} / 2+T)<1$, which implies that $F_{j_{L}}(-\bar{c} / 2+T)=1$. This is due to the fact that there is a strictly positive probability that $i$ waits until $T$, and, as in the case of $F_{i}$ above, $F_{j_{L}}$ must be continuous on $(0, T)$. However, as $F_{i}$ is constant in $(-\bar{c} / 2+T, T)$ and $c_{L}<\bar{c}, j_{L}$ strictly prefers $t_{j_{L}}=-\bar{c} / 2+T$ to all $t_{j_{L}}>-\bar{c} / 2+T$, and therefore $F_{j_{L}}(-\bar{c} / 2+T)<1$ contradicts the nonexistence of interior mass points.

With $(11), \min \left(b_{i}, b_{j_{L}}\right)=0$, and $F_{j_{L}}(-\bar{c} / 2+T)=1$, we get

$$
b_{i}=0 \text { and } b_{j_{L}}=\frac{1}{p_{L}}\left[1-\left(1-p_{L}\right) \exp \left(-\frac{1}{2}+\frac{T}{\bar{c}}\right)\right] .
$$

$b_{j_{L}}$ is strictly decreasing in $T$ with $\lim _{T \downarrow \bar{c} / 2} b_{j_{L}}=1$ and $\lim _{T \uparrow \bar{c} / 2-\bar{c} \ln p_{H}} b_{j_{L}}=0$. Hence, $\bar{c} / 2<T<\bar{c} / 2-\bar{c} \ln p_{H}$ is a necessary condition for the existence of a mixed strategy equilibrium. ${ }^{23}$

Step 2: It remains to show that (10), (11) and (12) indeed constitute an equilibrium. Consider first individual $i$ and suppose that $j$ follows $F_{j_{L}}$ and $F_{j_{H}}$, respectively. For any $t_{i} \in(0,-\bar{c} / 2+T], i$ 's expected payoff is

$$
v-p_{L} \int_{0}^{t_{i}} x \frac{1-p_{L}}{p_{L}} \frac{1}{\bar{c}} e^{-\frac{1}{2}+\frac{T-x}{\bar{c}}} d x-\left(1-p_{L}\right) e^{-\frac{1}{2}+\frac{T-t_{i}}{\bar{c}}}\left(\bar{c}+t_{i}\right)
$$

which is equal to $v-\left(1-p_{L}\right) \exp (-1 / 2+T / \bar{c}) \bar{c}$. If $i$ concedes in $T$, he gets

$$
v-p_{L} \int_{0}^{-\bar{c} / 2+T} x \frac{1-p_{L}}{p_{L}} \frac{1}{\bar{c}} e^{-\frac{1}{2}+\frac{T-x}{\bar{c}}} d x-\left(1-p_{L}\right)\left(\frac{\bar{c}}{2}+T\right)
$$

\footnotetext{
${ }^{23}$ To be precise, if $T=\bar{c} / 2-\bar{c} \ln p_{H}$, we get $b_{j_{L}}=0$ and $b_{i} \geq 0$ is not uniquely determined. Hence, there exists a continuum of mixed strategy equilibria where $i$ 's payoff is $v-\bar{c}$, as in the pure strategy equilibrium. We omit this case in order to simplify the exposition.
} 
which again is equal to $v-\left(1-p_{L}\right) \exp (-1 / 2+T / \bar{c}) \bar{c}$. Hence, $i$ is indifferent to all $t \in(0,-\bar{c} / 2+T] \cup\{T\}$. Any $t_{i} \in(-\bar{c} / 2+T, T)$ leads to a lower payoff.

Now turn to $j$ and suppose that $i$ follows $F_{i}$. The equilibrium strategy of $j_{H}$ follows from Lemma 1 . For $j_{L}$, a concession in $t \in[0,-\bar{c} / 2+T]$ yields an expected payoff of

$$
\int_{0}^{t}(v-x) \frac{1}{c_{L}} e^{-\frac{x}{c_{L}}} d x+e^{-\frac{t}{c_{L}}}\left(v-c_{L}-t\right)=v-c_{L} .
$$

Hence, $j_{L}$ is indeed indifferent to all $t \in[0,-\bar{c} / 2+T]$. For all $t>-\bar{c} / 2+T, j_{L}$ 's expected payoff is strictly lower. The ex ante expected payoffs in the second row of (6) and (7) follow directly from these calculations.

\section{A.4 Proof of Lemma 4}

By Assumption 1 and Lemma $1, F_{i_{H}}(t)=0$ for all $t<T$ and $F_{i_{H}}(t)=1$ otherwise, $i=1,2$. Thus, for $i_{L}$, a concession in $T$ is strictly preferred to any $t \in\left(-c_{L} / 2+T, T\right)$. Suppose that $j_{L}$ follows $F_{j_{L}}$. For any $t_{i} \in[0, \bar{t}), i_{L}$ 's payoff is

$$
p_{L} \int_{0}^{t_{i}}(v-x) \frac{1}{p_{L} c_{L}} e^{-\frac{x}{c_{L}}} d x+e^{-\frac{t_{i}}{c_{L}}}\left(v-t_{i}-c_{L}\right)=v-c_{L} .
$$

By choosing $t_{i}=T, i_{L}$ gets

$$
\begin{aligned}
& p_{L} \int_{0}^{\bar{t}}(v-x) \frac{1}{p_{L} c_{L}} e^{-\frac{x}{c_{L}}} d x+e^{-\frac{\bar{t}}{c_{L}}}\left(v-T-\frac{c_{L}}{2}\right) \\
= & v-c_{L}+\exp \left(-\frac{\bar{t}}{c_{L}}\right)\left(\bar{t}-T+\frac{c_{L}}{2}\right) .
\end{aligned}
$$

If

$$
-\frac{c_{L}}{2}+T<-c_{L} \ln \left(1-p_{L}\right)
$$

$\bar{t}=-\frac{c_{L}}{2}+T$, and $i_{L}$ is indifferent between all $t_{i} \in[0, \bar{t}) \cup\{T\}:$ (13) implies that $F_{i_{L}}(\bar{t})<1$ and $i_{L}$ waits until $T$ with strictly positive probability. If (13) is violated, 
$\bar{t}=-c_{L} \ln \left(1-p_{L}\right)$ and $F_{i_{L}}(\bar{t})=1$, that is, $i_{L}$ concedes with probability one before

$\bar{t}<T$. Indeed, waiting until $T$ would lead to a payoff lower than $v-c_{L}$. Since any symmetric equilibrium must be in mixed strategies, this is the only symmetric equilibrium. Expected payoff of $i_{L}$ is $v-c_{L}$, and expected payoff of $i_{H}$ is

$$
\begin{aligned}
& p_{L} \int_{0}^{\bar{t}}(v-x) \frac{1}{p_{L} c_{L}} e^{-\frac{x}{c_{L}}} d x+e^{-\frac{\bar{t}}{c_{L}}}\left(v-T-\frac{c_{H}}{2}\right) \\
= & v-c_{L}+\exp \left(-\frac{\bar{t}}{c_{L}}\right)\left(c_{L}-\frac{c_{H}}{2}+\bar{t}-T\right) .
\end{aligned}
$$

Hence, the ex ante expected payoff is

$$
E\left(\pi_{i}\right)=v-c_{L}+p_{H} \exp \left(-\frac{\bar{t}}{c_{L}}\right)\left(c_{L}-\frac{c_{H}}{2}+\bar{t}-T\right) .
$$

Inserting $\bar{t}$ leads to (8).

\section{A.5 Proof of Lemma 5}

(i) Suppose that $T<\bar{c} / 2$. Together with (3) and (5),

$$
V_{i}^{N}=-p_{L} c_{L}-p_{H}\left(\frac{c_{H}}{2}+T\right)+\frac{\bar{c}}{2}+T=-p_{L} \frac{c_{L}}{2}+\left(1-p_{H}\right) T>0 .
$$

If $T>\bar{c} / 2$, expected payoff is $v-\bar{c}$ in case $(N, N)$ which is the payoff an informed individual $i$ can ensure by conceding immediately for both possible contribution costs. Since for a high contribution cost, $i$ strictly prefers waiting until $T$, his payoff must be strictly higher. Thus, $V_{i}^{\sigma_{j}=N}>0$ for all $T \in\left(c_{L} / 2, c_{H} / 2\right)$.

(ii) If $T<c_{L} / 2-c_{L} \ln p_{H}$, subtracting the first row in (4) from the first row in (8) leads to

$$
V_{i}^{I}(T)=-c_{L}-\frac{p_{H}}{2}\left(c_{H}-c_{L}\right) e^{\frac{1}{2}-\frac{T}{c_{L}}}+p_{H}\left(\frac{\bar{c}}{2}+T\right) .
$$


For $T \rightarrow c_{L} / 2,(14)$ converges to

$$
-c_{L}-\frac{p_{H}}{2}\left(c_{H}-c_{L}\right)+\frac{p_{H}}{2}\left(\bar{c}+c_{L}\right)=-p_{L} c_{L}-\frac{p_{H}}{2}\left(c_{H}-\bar{c}\right)<0 .
$$

Moreover, deriving (14) with respect to $T$ yields

$$
\frac{\partial V_{i}^{I}(T)}{\partial T}=\frac{1}{2} \frac{p_{H}}{c_{L}}\left(c_{H}-c_{L}\right) e^{\frac{1}{2}-\frac{T}{c_{L}}}+p_{H}>0 .
$$

If $c_{L} / 2-c_{L} \ln p_{H}<T<\bar{c} / 2$, using again (4) and (8) we have

$$
V_{i}^{I}(T)=-c_{L}-p_{H}^{2}\left(T+\frac{c_{H}}{2}-c_{L}+c_{L} \ln p_{H}\right)+p_{H}\left(\frac{\bar{c}}{2}+T\right)
$$

and

$$
\frac{\partial V_{i}^{I}(T)}{\partial T}=-p_{H}^{2}+p_{H}>0 .
$$

(iii) With (4), $i$ gets $v-\bar{c}$ if he does not become informed. By the same argument as in (i) for $T>\bar{c} / 2, i$ 's ex ante payoff in case $(I, I)$ must be strictly larger than $v-\bar{c}$, and thus $V_{i}^{I}>0$ for all $T \in\left(\bar{c} / 2, c_{H} / 2\right)$.

(iv) In (ii), monotonicity has been shown for $T<\bar{c} / 2$. Now suppose that $T>$ $\bar{c} / 2$. Consider first the case where $T$ is smaller than $c_{L} / 2-c_{L} \ln p_{H}$, i.e. $T \in$ $\left(\bar{c} / 2, c_{L} / 2-c_{L} \ln p_{H}\right)$. (For a sufficiently large $p_{H}$, this interval is empty.) Then, with (6) and (8),

$$
V_{i}^{I}(T)=-c_{L}-\frac{p_{H}}{2}\left(c_{H}-c_{L}\right) e^{\frac{1}{2}-\frac{T}{c_{L}}}+p_{H} \bar{c} e^{-\frac{1}{2}+\frac{T}{\bar{c}}}
$$

and

$$
\frac{\partial V_{i}^{I}(T)}{\partial T}=\frac{p_{H}}{2 c_{L}}\left(c_{H}-c_{L}\right) e^{\frac{1}{2}-\frac{T}{c_{L}}}+p_{H} e^{-\frac{1}{2}+\frac{T}{\bar{c}}}>0
$$

Now suppose $T$ is larger than $c_{L} / 2-c_{L} \ln p_{H}$, but smaller than $\bar{c} / 2-\bar{c} \ln p_{H}$, i.e. $T \in\left(c_{L} / 2-c_{L} \ln p_{H}, \min \left\{\bar{c} / 2-\bar{c} \ln p_{H}, c_{H} / 2\right\}\right)$. (This interval may be empty for a 
small $\left.p_{H}.\right)$ We get

$$
V_{i}^{I}(T)=-c_{L}-p_{H}^{2}\left(T+\frac{c_{H}}{2}-c_{L}+c_{L} \ln p_{H}\right)+p_{H} \bar{c} e^{-\frac{1}{2}+\frac{T}{\bar{c}}}
$$

and hence

$$
\frac{\partial V_{i}^{I}(T)}{\partial T}=-p_{H}^{2}+p_{H} e^{-\frac{1}{2}+\frac{T}{\bar{c}}}>-p_{H}^{2}+p_{H}>0 .
$$

Continuity of $V_{i}^{I}$ follows directly from continuity of the expected payoffs.

\section{A.6 Proof of Proposition 1}

From Lemma 5(i), it follows that the best response to $\sigma_{j}=N$ is to become informed. Now suppose that in case $(N, I)$ the pure strategy equilibrium is selected. With Lemma 5(ii)-(iii), there exists a unique $\tilde{T} \leq \bar{c} / 2$ such that the best response to $\sigma_{j}=I$ is to remain uninformed if and only if $T<\tilde{T}$. In this case, there are two asymmetric equilibria where one individual acquires information and the other individual remains uninformed. In addition, there is a symmetric equilibrium where the individuals randomize their information choice and learn their provision cost with probability $V_{i}^{N} /\left(V_{i}^{N}-V_{i}^{I}\right) \in(0,1)$. If $T>\tilde{T}$, there is a unique equilibrium where both individuals find out about their provision cost.

For the mixed strategy equilibrium in case $(N, I)$, this result follows from monotonicity of $V_{i}^{I}$ (Lemma $\left.5 \mathrm{ii}+\mathrm{iv}\right)$. Note that $\tilde{T}<\bar{c} / 2-\bar{c} \ln p_{H}$ as, for $T \rightarrow \bar{c} / 2-\bar{c} \ln p_{H}$, $V_{i}^{I}$ converges to the value of information in the pure strategy equilibrium and hence is strictly positive. ${ }^{24}$ Therefore, whenever $\bar{c} / 2-\bar{c} \ln p_{H}<c_{H} / 2$, there exists an interior $\tilde{T} \in\left(c_{L}, \bar{c} / 2-\bar{c} \ln p_{H}\right)$ such that the best response to $\sigma_{j}=I$ is to remain uninformed if and only if $T<\tilde{T}$, and information acquisition is strictly dominant if $T>\tilde{T}$. If $\bar{c} / 2-\bar{c} \ln p_{H}>c_{H} / 2$, the interval where in equilibrium both individuals acquire information can be empty which is the case if $\lim _{T \rightarrow c_{H} / 2} V_{i}^{I}$ is negative.

\footnotetext{
${ }^{24}$ This follows from the convergence of $i$ 's expected payoff in case $(N, I)$ for $T \rightarrow \bar{c} / 2-\bar{c} \ln p_{H}$.
} 


\section{A.7 Proof of Corollary 1}

Part (i) follows from Lemma 5(iii). For part (ii), the threshold $\tilde{T}$ is larger than $\bar{c} / 2$ if and only if the value of information is negative as $T$ approaches $\bar{c} / 2$. Moreover, $V_{i}^{I}$ may even be negative for all $T$. Note that $\bar{c} / 2-\bar{c} \ln p_{H}>c_{L} / 2-c_{L} \ln p_{H}$, and for small $p_{H}$, we have $c_{L} / 2-c_{L} \ln p_{H}>c_{H} / 2$. With monotonicity of (16), it follows that, for all $T \in\left(c_{L} / 2, c_{H} / 2\right), V_{i}^{I}$ is smaller than

$$
\lim _{T \rightarrow c_{H} / 2} V_{i}^{I}(T)=-c_{L}-\frac{p_{H}}{2}\left(c_{H}-c_{L}\right) e^{\frac{1}{2}-\frac{c_{H}}{2 c_{L}}}+p_{H} \bar{c} e^{-\frac{1}{2}+\frac{c_{H}}{2 \bar{c}}}
$$

which is negative for small $p_{H}$ since the second and the third term approach zero if $p_{H} \rightarrow 0$. For intermediate values of $p_{H}$, we have $\tilde{T} \in\left(\bar{c} / 2, c_{H} / 2\right)$.

\section{A.8 Proof of Proposition 2}

Suppose that $i$ remains uninformed and $j$ acquires information. If $T<\bar{c} / 2$, $i$ 's expected payoff is strictly higher than $j$ 's expected payoff (compare the first row in (6) to the first row in (7)). Thus, if $T<\min \{\bar{c} / 2, \tilde{T}\}$, in the equilibrium of the game of sequential information acquisition, the first mover remains uninformed.

If $T>\bar{c} / 2$ and in case $(N, I)$ the mixed strategy equilibrium is selected, by continuity of the expected payoffs, $E\left(\pi_{i}\right)>E\left(\pi_{j}\right)$ also holds for $T=\bar{c} / 2+\delta, \delta>0$ sufficiently small (compare the second rows in (6) and (7)). As $T \rightarrow \bar{c} / 2-\bar{c} \ln p_{H}$, the payoff of the uninformed player $i$ approaches $v-\bar{c}$, while the informed player $j$ gets strictly more than $v-\bar{c} .^{25}$ The difference in payoffs $E\left(\pi_{j}\right)-E\left(\pi_{i}\right)$ is strictly increasing in $T$, and there is a critical value $T_{s}$ where $E\left(\pi_{j}\right)=E\left(\pi_{i}\right)$. Depending on the parameters of the model, $T_{s}$ can be smaller or larger than the threshold $\tilde{T}$ for information acquisition. If $T_{s}>\tilde{T}$, in all equilibria where one player chooses to remain uninformed, this will be the first mover. If $T_{s}<\tilde{T}$, there can also be equilibria of the game with sequential information acquisition where the first mover chooses to acquire information and the second mover remains uninformed. ${ }^{26}$

\footnotetext{
${ }^{25}$ By choosing $q_{j_{L}}(0)=1$ and $q_{j_{H}}(T)=1, j$ can ensure a payoff of at least $v-p_{L} c_{L}-$ $p_{H}\left(c_{H} / 2+T\right)$, which is strictly larger than $v-\bar{c}$; thus, his equilibrium payoff cannot be smaller.

${ }^{26}$ This is the case, for instance, if $c_{L}=2, c_{H}=10$, and $p_{H}=0.3$. There, in case $(N, I)$,
} 


\section{A.9 Proof of Proposition 3}

First of all, we solve for the payoffs in the war of attrition. In case $(N, N)$, expected payoffs are as in (3). In the symmetric equilibrium in case $(I, I)$, if it is common knowledge that both have a low cost, both individuals randomize and get an expected payoff of $v-c_{L}$. If both have a high cost, they wait until $T$. This leads to an ex ante expected payoff of

$$
v-p_{L} c_{L}-p_{H}\left(T+\frac{c_{H}}{2}\right)
$$

In case $(N, I)$, if $T<\bar{c} / 2$, in equilibrium of the war of attrition, we have $q_{i}(T)=$ $q_{j_{H}}(T)=1$ and $q_{j_{L}}(0)=1$. The uninformed individual $i$ gets an expected payoff of

$$
v-p_{H}\left(T+\frac{c_{H}}{2}\right)
$$

which is strictly larger than (18). Thus, the best response to information acquisition of $j$ is to remain uninformed. The informed individual gets

$$
v-p_{L} c_{L}-p_{H}\left(T+\frac{c_{H}}{2}\right)
$$

which is strictly larger than the payoff in case $(N, N)$. Therefore, in the equilibrium of the game of information acquisition, only one individual acquires information.

For $T \geq \bar{c} / 2$, the same is true if in case $(N, I)$ the equilibrium with $q_{j_{L}}(0)=1$ is selected; then, in the war of attrition, equilibrium payoffs are as above. If in case $(N, I)$ the equilibrium with $q_{i}(0)=1$ is selected, the uninformed individual gets an expected payoff of $v-\bar{c}$; in the game of information acquisition, both individuals acquire information.

$\left.\overline{E\left(\pi_{j}\right)>E\left(\pi_{i}\right) \text { in some interval }(\tilde{T}-\delta}, \tilde{T}\right), \delta>0$. 


\section{A.10 Proof of Proposition 4}

First, consider the war of attrition fixing the decisions on information. We only analyze the case where $T<-(v-\bar{c})$. Note that this implies that, in the war of attrition, uninformed individuals prefer to wait until $T$. Thus, in case $(N, N)$ where no individual is informed, both get an expected payoff of $-T$. Moreover, if individual $i$ is uninformed and individual $j$ knows his cost of provision (case $(N, I)$ ), $i$ and $j_{H}$ prefer waiting until $T$ to any concession before $T$. Since $v-c_{L}>-T, j_{L}$ will concede immediately. Ex ante expected payoffs in case $(N, I)$ are

$$
\begin{aligned}
& E\left(\pi_{i}\right)=p_{L} v+\left(1-p_{L}\right)(-T) \\
& E\left(\pi_{j}\right)=p_{L}\left(v-c_{L}\right)+\left(1-p_{L}\right)(-T)
\end{aligned}
$$

Now consider the war of attrition if both individuals know their provision cost. Due to Assumption 1' on the time limit, there is no equilibrium where an individual concedes immediately or waits until $T$ for both types. In the symmetric equilibrium, $q_{i_{H}}(T)=q_{j_{H}}(T)=1$, and low types randomize according to $F_{i_{L}}(t)=\frac{1}{p_{L}} \Phi\left(t ; c_{L}, \bar{t}, 0\right)$ where $\bar{t}=\min \left\{T-\left(-v+c_{L}\right),-c_{L} \ln p_{H}\right\}$. Similar to Lemma 4, if $T$ is small, lowcost types put a mass point at $T$, and if $T$ is large, they concede before $T$ with probability one. ${ }^{27}$ This leads to an ex ante expected payoff in case $(I, I)$ equal to

$E\left(\pi_{i}\right)= \begin{cases}v-c_{L}, & T<-\left(v-c_{L}\right)-c_{L} \ln p_{H} \\ \left(1-p_{H}^{2}\right)\left(v-c_{L}\right)-p_{H}^{2}\left(c_{L} \ln p_{H}+T\right), & T \geq-\left(v-c_{L}\right)-c_{L} \ln p_{H}\end{cases}$

In the game of information acquisition, suppose that $j$ acquires information and $T<-\left(v-c_{L}\right)-c_{L} \ln p_{H}$. If $i$ acquires information, his expected payoff is $v-c_{L}$ (compare (20)). If $i$ remains uninformed, by (19), he gets an expected payoff of

\footnotetext{
${ }^{27} \mathrm{~A}$ proof that these strategies constitute an equilibrium is omitted.
} 
$p_{L} v+\left(1-p_{L}\right)(-T)$ which is strictly larger than

$$
\begin{aligned}
& p_{L} v+\left(1-p_{L}\right)\left(\left(v-c_{L}\right)+c_{L} \ln p_{H}\right) \\
= & v-c_{L}+c_{L}\left(1-p_{H}+p_{H} \ln p_{H}\right) \\
> & v-c_{L}
\end{aligned}
$$

where the inequality follows from the fact that $1-p_{H}+p_{H} \ln p_{H}>0$ for all $p_{H} \in(0,1)$.

Thus, if $T$ is sufficiently small, only one individual acquires information. For $T \geq-\left(v-c_{L}\right)-c_{L} \ln p_{H}$, by continuity of the expected payoff in (20), the same result holds if $T$ is sufficiently close to $-\left(v-c_{L}\right)-c_{L} \ln p_{H}$. For larger $T$, equilibrium information acquisition depends on the parameter values. ${ }^{28}$

\section{References}

[1] Amann, E., Leininger, W., 1996. Asymmetric all-pay auctions with incomplete information: the two-player case. Games and Economic Behavior 14, 1-18.

[2] Bilodeau, M., Slivinski, A., 1996. Toilet cleaning and department chairing: volunteering a public service. Journal of Public Economics 59, 299-308.

[3] Bishop, D.T., Cannings, C., 1978. A generalized war of attrition. Journal of Theoretical Biology 70, 85-124.

[4] Bliss, C., Nalebuff, B., 1984. Dragon-slaying and ballroom dancing: the private supply of a public good. Journal of Public Economics 25, 1-12.

[5] Bulow, J., Klemperer, P., 1999. The generalized war of attrition. American Economic Review 89, 175-189.

[6] Che, Y.-K., Gale, I., 1998. Caps on political lobbying. American Economic Review 88, 643-651.

\footnotetext{
${ }^{28}$ Note that, while both (19) and (20) are decreasing in $T$, the slope of (19) is steeper. As in Corollary 1 , whenever $p_{H}$ is small, it is more likely that only one individual will acquire information in equilibrium.
} 
[7] Crémer, J., 1995. Arm's length relationships. Quarterly Journal of Economics 110, 275-295.

[8] Fudenberg, D., Tirole, J., 1986. A theory of exit in duopoly. Econometrica 54, 943-960.

[9] Fudenberg, D., Tirole, J., 1991. Game Theory. Cambridge (Mass.): MIT Press.

[10] Ghemawat, P., Nalebuff, B., 1985. Exit. Rand Journal of Economics 16, 184-194.

[11] Ghemawat, P., Nalebuff, B., 1990. The devolution of declining industries. Quarterly Journal of Economics 105, 167-186.

[12] Hendricks, K., Weiss, A., Wilson, C., 1988. The war of attrition in continuous time with complete information. International Economic Review 29, 663-680.

[13] Hernando-Veciana, A., 2009. Information acquisition in auctions: sealed bids vs. open bids. Games and Economic Behavior 65, 372-405.

[14] Hernando-Veciana, A., Tröge, M., 2010. The insider's curse. Games and Economic Behavior, forthcoming.

[15] Kessler, A.S., 1998. The value of ignorance. RAND Journal of Economics 29, 339-354.

[16] Krishna, V., Morgan, J., 1997. An analysis of the war of attrition and the all-pay auction. Journal of Economic Theory 72, 343-362.

[17] LaCasse, C., Ponsati, C, Barham, V., 2002. Chores. Games and Economic Behavior 39, 237-281.

[18] Larson, N., 2009. Private value perturbations and informational advantage in common value auctions. Games and Economic Behavior 65, 430-460.

[19] Maynard Smith, J., 1974. The theory of games and the evolution of animal conflicts. Journal of Theoretical Biology 47, 209-219. 
[20] Milgrom, P., Weber, R., 1982. The value of information in a sealed-bid auction. Journal of Mathematical Economics 10, 105-114.

[21] Morath, F., 2010. Strategic information acquisition and the mitigation of global warming. Journal of Environmental Economics and Management 59, 206-217.

[22] Morath, F., Münster, J., 2010. Information acquisition in conflicts. SFB/TR 15 Discussion Paper No. 314.

[23] Myatt, D.P., 2005. Instant exit from the asymmetric war of attrition. University of Oxford, Discussion Paper 160.

[24] Otsubo, H, Rapoport, A., 2008. Dynamic volunteer's dilemmas over a finite time horizon: an experimental study. Journal of Conflict Resolution 52, 961-984.

[25] Riley, J.G., 1980. Strong evolutionary equilibrium and the war of attrition. Journal of Theoretical Biology 82, 383-400.

[26] Riley, J.G., 1999. Asymmetric contests: a resolution of the Tullock paradox. In: Howitt, P., De Antoni, E.,Leijonhufvud, A. (eds.) Money, markets and method: essays in honor of Robert W. Clower, 190-207.

[27] Sahuguet, N., 2006. Volunteering for heterogeneous tasks. Games and Economic Behavior 56, 333-349. 